\title{
INSIGHTS GAINED FROM WEAKLY $P$ PROPERTIES INTO EQUIVALENCES OF SEPARATION AXIOMS
}

\section{CHARLES DORSETT}

Department of Mathematics

Texas A\&M University-Commerce

Commerce, Texas 75429

USA

e-mail: charles.dorsett@tamuc.edu

\begin{abstract}
In 1975 , it was proven that a space is $R_{1}$ iff its $T_{0}$-identification space is Hausdorff. The 1975 work motivated the introduction and investigation of weakly $P o$ properties, which led to the introduction and investigation of weakly $P 1$ and weakly $P 2$ properties. Within recent papers, it was established that in weakly $P 1$ spaces $T_{0}$ and $T_{1}$ are equivalent, and in weakly $P 2$ spaces $T_{0}, T_{1}$, and $T_{2}$ are equivalent. Within this paper, infinitely many topological properties, in addition to weakly $P 1$ or weakly $P 2$, are given for each weakly $P 1$ or weakly $P 2$ for which each of $T_{0}$ and $T_{1}$ or $T_{0}, T_{1}$, and $T_{2}$ are equivalent, including the least of all such topological property in each of the two cases.
\end{abstract}

\section{Introduction and Preliminaries}

$T_{0}$-identification spaces were introduced in 1936 [11].

Definition 1.1. Let $(X, T)$ be a space, $R$ be the equivalence relation on $X$ defined by $x R y$ iff $C l(\{x\})=C l(\{y\}), X_{0}$ be the set of $R$ equivalence 2010 Mathematics Subject Classification: 54A05, 54B15, 54D10.

Keywords and phrases: topological properties, $T_{0}$-identification spaces, weakly $P$.

Received July 15, 2016

(C) 2016 Scientific Advances Publishers 
classes of $X, N: X \rightarrow X_{0}$ be the natural map, and $Q(X, T)$ be the decomposition topology on $X_{0}$ determined by $(X, T)$ and the natural map $N$. Then $\left(X_{0}, Q(X, T)\right)$ is the $T_{0}$-identification space of $(X, T)$ [11].

Within the 1975 paper [10], weakly Hausdorff was characterized using $T_{0}$-identification spaces.

Theorem 1.1. A space $(X, T)$ is weakly Hausdorff iff its $T_{0}$-identification space is Hausdorff [10].

In the 2015 paper [2], the question of whether $T_{0}$-identification spaces could be used to uniquely define other weakly $P$ properties behaving in the same manner as weakly Hausdorff led to the introduction and investigation of weakly Po properties.

Definition 1.2. Let $P$ be a topological property for which $P o=(P$ and $\left.T_{0}\right)$ exists. Then $(X, T)$ is weakly $P o$ iff its $T_{0}$-identification space $\left(X_{0}, Q(X, T)\right)$ has property $P$. A topological property $P o$ for which weakly $P o$ exists is called a weakly $P o$ property [2].

In the 2015 paper [2], it was proven that for a topological property $P$ for which weakly $P o$ exists, weakly $P o$ is a unique, topological property. In addition, since for each space $(X, T),\left(X_{0}, Q(X, T)\right)$ is $T_{0}$ [11], then, as given in the 2015 paper [2], a space is weakly $P_{0}$ iff its $T_{0}$-identification space has property $P o$.

Within the 1975 paper [10], it was proven that weakly Hausdorff is equivalent to the $R_{1}$ separation axiom, which was introduced in 1961 [1].

Definition 1.3. A space $(X, T)$ is $R_{1}$ iff for $x, y \in X$, such that $C l(\{x\}) \neq C l(\{x\})$, there exist disjoint open sets $U$ and $V$ such that $x \in U$ and $y \in V[1]$.

In the 1961 paper [1], the following characterizations of $T_{2}$ were given: For a space $(X, T)$, the following are equivalent: (a) $(X, T)$ is $T_{2}$, (b) $(X, T)$ is $\left(R_{1}\right.$ and $\left.T_{1}\right)$, and (c) $(X, T)$ is $\left(R_{1}\right.$ and $\left.T_{0}\right)$. 
Also, within the 1961 paper [1], the $R_{0}$ separation axiom was rediscovered and used to further characterize $T_{1}$ spaces.

Definition 1.4. A space $(X, T)$ is $R_{0}$ iff for each open set $O$ and each $x \in O, C l(\{x\}) \subseteq O$.

In the 1961 paper [1], it was shown that $R_{1}$ implies $R_{0}$ and a space is $T_{1}$ iff it is $\left(R_{0}\right.$ and $\left.T_{0}\right)$.

Within weakly $P_{0}$ properties, the $T_{0}$ separation axiom has a major role. Thus the question of what would happen if $T_{0}$ in the definition of weakly $P_{o}$ properties was replaced by $T_{1}$ or by $T_{2}$ arose leading to the introduction and investigation of weakly $P 1$ and weakly $P 2$ properties.

Definition 1.5. Let $P$ be a topological property for which $P 1=(P$ and $\left.T_{1}\right)$ exists. Then a space $(X, T)$ is weakly $P 1$ iff its $T_{0}$-identification space $\left(X_{0}, Q(X, T)\right)$ has property $P 1[3]$.

Definition 1.6. Let $P$ be a topological property for which $P 2=(P$ and $\left.T_{2}\right)$ exists. Then a space $(X, T)$ is weakly $P 2$ iff its $T_{0}$-identification space $\left(X_{0}, Q(X, T)\right)$ has property $P 2$ [4].

The continued investigation of weakly $P 1$ properties [5] has revealed that for weakly $P 1$ spaces, $T_{0}$ and $T_{1}$ are equivalent and for weakly $P 2$ spaces, $T_{0}, T_{1}$, and $T_{2}$ are all equivalent [6]. Thus, the question of whether there are additional topological properties for which $T_{0}$ and $T_{1}$ are equivalent and for which $T_{0}, T_{1}$, and $T_{2}$ are all equivalent arises. The equivalences given above were not expected, but, in this case, as is often true, discoveries lead to additional questions for consideration and hopefully resolution. Below for each weakly $P 1$ property and for each weakly $P 2$ property, infinitely many related topological properties are given in which the equivalences above hold. 


\section{Related Topological Properties for a Weakly $P 1$ and Weakly P2 Property Preserving the Equivalences}

Confronted by a new problem, mathematicians are trained to search for a way to relate the new problem to an already solved problem and, if successful, use the solution of the solved problem to aid in the solution of the new problem. For the cited new problems above, there are solved problems that can be applied to greatly aid in their resolution.

Within the 2016 paper [7], the following result was proven: "Let $Q$ be a topological property for which weakly Qo exists and let $\mathcal{S}=\{S o \mid S$ is a topological property, So exists, and $S o$ implies $Q o\}$. Then (weakly $Q o$ )o $\in \mathcal{S}$, for each weakly $P o$ property $W$ such that Wo implies Qo, (weakly $W o$ )o $\in \mathcal{S}$, each element of $\mathcal{S}$ implies weakly $Q o$, and there exists the topological property $Q_{\min }=\left((\right.$ weakly $Q o)$ or "not- $T_{0}$ "), where "not- $T_{0}$ " is the negation of $T_{0}$, weaker than weakly $Q o$ such that $\left(Q_{\min }\right) o \in \mathcal{S}$ ". In the weakly $P 1$ paper [3], it was shown that for a weakly $P 1$ property $Q 1, Q 1$ is a weakly $P o$ property, i.e., $Q 1=(Q 1) o$, which is combined with the results above to quickly establish the following useful results in the resolution of the new problems above.

Corollary 2.1. Let $Q$ be a topological property for which weakly $Q 1$ exists and let $\mathcal{S}(Q 1)=\{S o \mid S$ is a topological property, So exists, and So implies $Q 1\}$. Then $\mathcal{S}(Q 1)=\{S o \mid S$ is a topological property, So exists, and So implies $(Q 1) o\}$.

Corollary 2.2. Let $Q$ be a topological property for which weakly $Q 1$ exists and let $\mathcal{S}(Q 1)=\{S o \mid S$ is a topological property, So exists, and So implies $Q 1\}$. Then (weakly $Q 1) o \in \mathcal{S}(Q 1)$, for each weakly Po property $W$ such that Wo implies $Q 1,($ weakly Wo o $\in \mathcal{S}(Q 1)$, each element of $\mathcal{S}(Q 1)$ implies weakly $Q 1$, and there exists the topological property $\left(Q_{\min }\right) 1=\left((\right.$ weakly $Q 1)$ or "not- $T_{0}$ ") weaker than weakly $Q 1$ such that $\left(\left(Q_{\min }\right) 1\right) o \in \mathcal{S}(Q 1)$. 
Also, in the 2016 paper [7], it was established that for a topological property for which weakly $P_{0}$ exists, $P_{\min }$ is the least topological property for which a space has property $P_{0}$ iff it has property $\left(P_{\min }\right.$ and $\left.T_{0}\right)$, giving the next result.

Corollary 2.3. Let $P$ be a topological property for which weakly $P 1$ exists. Then $\left(P_{\min }\right) 1$ is the least topological property for which a space has property $P 1$ iff it has properties $\left(\left(P_{\min }\right) 1\right.$ and $\left.T_{0}\right)$.

Within the second 2016 paper [8], for a weakly Po property Qo, the special role played by $Q_{\min }$ was used to give infinitely many topologically distinct, non-weakly $P o$ topological properties weaker than weakly $Q o$ and stronger than $Q_{\min }$, which together with $T_{0}$, are equivalent to $Q$, which will be combined with the results above to extend the 2016 result [8] to weakly $P 1$ properties.

Let $m$ and $n$ represent natural number greater than or equal to 2 .

Definition 2.1. Let $A(n)$ represent a set with $n$ distinct elements, $X$ be a set containing the elements of $A(n)$, and $T(A(n))$ be the topology on $X$ defined by $T(A(n))=\{B \subseteq X \mid A(n) \subseteq B$ or $B=\phi\}$ [8].

Definition 2.2. A space $(X, T)$ has property $T(n)$ iff there exists a subset $A(n)$ of $X$ such that $T=T(A(n))$ [8].

In the 2016 paper [8], it was shown that each $T(n)$ space is "not- $T_{0}$ " and not a weakly $P o$ property, that $Q(n)=($ weakly $Q o$ or $T(n))$ is a topological property weaker than weakly $Q o$ and stronger than $Q_{\min }$ such that a space has property $Q o$ iff it has property $\left(Q(n)\right.$ and $\left.T_{0}\right)$, and that for $m<n, Q(m)$ and $Q(n)$ are distinct topological properties, which is combined with the results above to give the next result. 
Corollary 2.4. For each $n$ and each weakly $P 1$ property $Q 1, Q(n) 1=$ ((weakly Q1) or $T(n))$ is a non-weakly P1 topological property weaker than weakly $Q 1$ and stronger than $\left(Q_{\min }\right) 1$ such that a space has property $Q 1$ iff it has property $\left((Q(n) 1)\right.$ and $\left.T_{0}\right)$.

Thus, there are infinitely many non-weakly $P 1$ topological properties $W$ weaker than weakly $Q 1$ and stronger than $\left(Q_{\min }\right) 1$ such that a space has property $Q 1$ iff it has property ( $W$ and $T_{0}$ ).

In the first 2016 paper [7], it was shown that for a weakly $P_{o}$ property $Q o, Q_{(\min , \max )}=\left((\right.$ weakly $Q o)$ and "not- $T_{0}$ ") also plays a special role: $Q_{(\min , \max )}$ is the least topological property weaker than $Q o$ and stronger than weakly $Q o$ such that a space is $Q o$ iff it is $\left(Q_{(\min , \max )}\right.$ and $\left.T_{0}\right)$. Combining this result with those above give the following result.

Corollary 2.5. Let $Q$ be a topological property for which weakly $Q 1$ exists. Then $\left(Q_{(\min , \max )}\right) 1=\left((\right.$ weakly $Q 1)$ and "not- $T_{0}$ ") is the least topological property weaker than $Q 1$ and stronger than weakly $Q 1$ such that a space is $Q 1$ iff it is $\left(\left(Q_{(\min , \max )}\right) 1\right.$ and $\left.T_{0}\right)$.

Within the second 2016 paper [8], for each weakly Po property Qo, $Q(1, n)$ was defined and used to give infinitely many more topological properties, which together with $T_{0}$, are equivalent to $Q_{0}$.

Definition 2.3. Let $Q$ be a topological property for which weakly $Q o$ exists. A space $(X, T)$ is $Q(1, n)$ iff it is weakly $Q o$, there exist $n$ distinct elements $a_{1}, \cdots, a_{n}$ all of whose closures are equal, and for all other $x \in X, C l(\{x\})=C l(\{y\})$ iff $x=y[8]$.

In the 2016 paper [8], for a weakly Po property $Q o$, it was shown that $Q(1, n)$ exists, $Q(1, n)$ is weaker than $Q o$ and stronger than $Q_{(\min , \max )}$, $Q o=\left(Q(1, n)\right.$ and $\left.T_{0}\right)$, and for each $Q o$ space $(Y, S)$ there are infinitely 
many topologically spaces all with topologically distinct topological properties that are weaker than $Q o$ and stronger than $Q_{(\min , \max )}$, which together with $T_{0}$, equals $Q o$ and all having a $T_{0}$-identification space homeomorphic to $(Y, S)$.

Definition 2.4. Let $Q$ be a topological property for which weakly $Q 1$ exists. A space $(X, T)$ is $(Q(1, n)) 1$ iff it is weakly $Q 1$, there exist $n$ distinct elements $a_{1}, \cdots, a_{n}$ all of whose closures are equal, and for all other $x \in X, C l(\{x\})=C l(\{y\})$ iff $x=y$.

Corollary 2.6. Let $Q$ be a topological property for which weakly $Q 1$ exists. Then $(Q(1, n)) 1$ exists, $(Q(1, n)) 1$ is weaker than $Q 1$ and stronger than $\left(Q_{(\min , \max )}\right) 1, Q 1=\left((Q(1, n)) 1\right.$ and $\left.T_{0}\right)$, and for each $Q 1$ space $(Y, S)$, there are infinitely many topologically spaces all with topologically distinct topological properties that are weaker than $Q 1$ and stronger than $\left(Q_{(\min , \max )}\right) 1$, which together with $T_{0}$, equals $Q 1$ and all having a $T_{0}$-identification space homeomorphic to $(Y, S)$.

As indicated in the paper [8], if $(Y, S)$ has property $Q_{o}$, where $Q_{o}$ is a weakly $P_{0}$ property, with $p$ or more elements, then $Q(1, n)$ can be extended to $Q\left(p, n_{1}, \cdots, n_{p}\right)$ that behaves in the same manner as $Q(1, n)$ and can be used to give many more topological properties weaker than $Q o$ and stronger than $Q_{(\min , \max )}$, which together with $T_{0}$, is equivalent to Qo. In the same manner as above, each of these new topological properties can be used to give a topological property weaker than $Q 1$ and stronger than $\left(Q_{(\min , \max )}\right) 1$, which together with $T_{0}$, is equivalent to $Q 1$.

Theorem 2.1. Let $P$ be a topological property such that ( $P$ and $T_{0}$ ) exists. Then ( $P$ and $T_{0}$ ) implies $P 1$ iff $T_{0}$ and $T_{1}$ are equivalent. 
Proof. Suppose $\left(P\right.$ and $\left.T_{0}\right)$ implies $P 1$. Thus, if $(X, T)$ is a $P$ space with property $T_{0}$, then $(X, T)$ has property $P 1$, which implies $(X, T)$ is $T_{1}$. Since $T_{1}$ implies $T_{0}$, then $T_{0}$ and $T_{1}$ are equivalent.

Clearly, the converse is true.

Corollary 2.7. Let $Q$ be a topological property for which weakly $Q 1$ exists. Then for each topological property $P$ given above for which $\left(P\right.$ and $\left.T_{0}\right)=Q 1, T_{0}$ and $T_{1}$ are equivalent.

Since for a topological property $Q$ for which weakly $Q 2$ exists, $Q 2=(Q 2) o[6]$, then each of the results above can be correctly restated by replacing weakly $Q 1$ by weakly $Q 2$ and $Q 1$ by $Q 2$ and since for a topological property $P$ for which $\left(P\right.$ and $\left.T_{0}\right)$ exists, $\left(P\right.$ and $\left.T_{0}\right)$ implies $P 2$ iff $T_{0}, T_{1}$, and $T_{2}$ are equivalent, then there are infinitely many known topologically distinct topological properties for which each of $T_{0}$, $T_{1}$, and $T_{2}$ are equivalent for each weakly $P 2$ property.

Of the many topological properties $P$ for which ( $P$ and $\left.T_{0}\right)$ exists and $T_{0}$ and $T_{1}$ are equivalent, is there a least such topological property and of the many topological properties $P$ for which $\left(P\right.$ and $\left.T_{0}\right)$ exists and $T_{0}, T_{1}$, and $T_{2}$ are equivalent, is there a least such topological property? These questions are resolved in the last section of this paper.

\section{The Least Topological Properties Preserving the Equivalences}

Theorem 3.1. The least of all topological properties $P$ for which ( $P$ and $\left.T_{0}\right)$ exists and $T_{0}$ and $T_{1}$ are equivalent is $\left(R_{0}\right.$ or "not- $T_{0}$ ").

Proof. Let $\mathcal{P} 1=\{S o \mid S$ is a topological property, So exists, and So implies $\left.T_{1}\right\}$. By the results above, ((weakly $T_{1}$ ) or "not- $T_{0}$ ") is the least topological property $P$ for which a space has property $T_{1}$ iff it is $\left(P\right.$ and $\left.T_{0}\right)$. Since weakly $T_{1}=$ weakly $\left(R_{0}\right) o[2]$, then $P=\left(\left(R_{0}\right)\right.$ or "not- $T_{0}$ ") is the 
least of all topological properties $P$ for which a space is $T_{1}$ iff it is ( $P$ and $\left.T_{0}\right)$. Thus $\left(\left(R_{0}\right)\right.$ or "not- $T_{0}$ ") is the least topological property $P$ for which $\left(P\right.$ and $\left.T_{0}\right)$ exists and $T_{0}$ and $T_{1}$ are equivalent.

Theorem 3.2. The least of all topological properties $P$ for which ( $P$ and $\left.T_{0}\right)$ exists and $T_{0}, T_{1}$, and $T_{2}$ are equivalent is $\left(\left(R_{1}\right)\right.$ or "not$\left.T_{0}{ }^{\prime}\right)$.

Proof. Let $\mathcal{P} 2=\{S o \mid S$ is a topological property, So exists, and So implies $\left.T_{2}\right\}$. By the results above, ((weakly $\left.T_{2}\right)$ or "not- $T_{0}$ ") is the least topological property $P$ for which a space has property $T_{2}$ iff it is $\left(P\right.$ and $\left.T_{0}\right)$. Since weakly $T_{2}=$ weakly $\left(R_{1}\right) o[2]$, then $P=\left(\left(R_{1}\right)\right.$ or "not- $T_{0}$ ") is the least of all topological properties $P$ for which a space is $T_{2}$ iff it is ( $P$ and $\left.T_{0}\right)$. Thus $\left(\left(R_{1}\right)\right.$ or "not- $T_{0}$ ") is the least topological property $P$ for which ( $P$ and $\left.T_{0}\right)$ exists and $T_{0}$ and $T_{1}$ are equivalent.

The last two results follow immediately from the results above.

Corollary 3.1. Let $(X, T)$ be $R_{0}$. Then $(X, T)$ is $T_{0}$ iff $(X, T)$ is $T_{1}$.

Corollary 3.2. Let $(X, T)$ be $R_{1}$. Then $(X, T)$ is $T_{0}$ iff $(X, T)$ is $T_{1}$ iff $(X, T)$ is $T_{2}$.

Thus, knowledge of solved problems that are related to the problems above and then applying that knowledge has made difficult problems quickly solvable. In the 1961 paper [1], the focus was the separation axioms $T_{1}$ and $T_{2}$. The introduction and investigation of weakly $P o$, weakly $P 1$, and weakly $P 2$ properties has changed the focus from $T_{1}$ and $T_{2}$ to $R_{0}, R_{1}$, and infinitely many new topological properties that add much and give greater insight into the working of mathematics. With the focus on $T_{1}$ and $T_{2}$ in 1961 [1], one characterization of $T_{1}$ was given and two characterizations of $T_{2}$ was given and, even though the knowledge 
was available, use of $R_{0}$ and $R_{1}$ in the equivalence questions above were overlooked. However, as a result of the 1961 paper [1] and the 1975 paper [10], today infinitely many topological properties are known for which the equivalences above are true and infinitely many new characterizations of $T_{1}$ and $T_{2}$ have been discovered [9].

\section{References}

[1] A. Davis, Indexed systems of neighborhoods for general topological spaces, Amer. Math. Monthly 68 (1961), 886-893.

[2] C. Dorsett, Weakly $P$ properties, Fundamental Journal of Mathematics and Mathematical Sciences 3(1) (2015), 83-90.

[3] C. Dorsett, Weakly $P 1$, weakly $P o$ and $T_{0}$-identification $P$ properties, Fundamental Journal of Mathematics and Mathematical Sciences 6(1) (2016), 33-43.

[4] C. Dorsett, Weakly P2 properties and related properties, Fundamental Journal of Mathematics and Mathematical Sciences 4(1) (2015), 11-21.

[5] C. Dorsett, Additional weakly $P 1$ properties and "not-(weakly $P 1)$ " properties, submitted.

[6] C. Dorsett, Weakly $P 2$ and weakly $P 1$ properties, submitted.

[7] C. Dorsett, Weakly $P$ corrections and new, fundamental topological properties and facts, Fundamental Journal of Mathematics and Mathematical Sciences 5(1) (2016), 11-20.

[8] C. Dorsett, Infinitely many topological property characterizations of weakly Po properties, Pioneer Journal of Mathematics and Mathematical Sciences 17(1) (2016), 23-31.

[9] C. Dorsett, Application of weakly $P$ properties giving infinitely many new characterizations of $T_{1}$ and $T_{2}$ spaces, Journal of Mathematical Sciences: Advances and Applications 39 (2016), 89-98.

[10] W. Dunham, Weakly Hausdorff spaces, Kyungpook Math. J. 15(1) (1975), 41-50.

[11] M. Stone, Application of Boolean algebras to topology, Mat. Sb. 1 (1936), 765-771. 